\title{
Empathie und moderne Medizin: Luxus oder Notwendigkeit?
}

Otto Knüsel

Stark gekürzte und adaptierte Fassung eines Vortrags anlässlich des reha Schweiz Kongresses $2011 \mathrm{am}$ 27. Oktober 2011 in Bern.

Korrespondenz: Dr. med. Otto Knüsel Römersteig Postfach 10 CH-7304 Maienfeld otto.knuesel[at]gmx.ch
Was bedeutet eigentlich Empathie? Empathie mit einem anderen Menschen bedeutet, sich in dessen Lage zu versetzen und mit ihm mitzufühlen, d.h. sich darüber klar zu werden, was der andere fühlt oder fühlen könnte, sowie die eigenen Gefühle zu erkennen und angemessen zu reagieren. Ein Lebewesen ist mit einem anderen empathisch, wenn es sich in dieses hineinfühlt, sich also vorstellt, es wäre das andere, beziehungsweise so fühlt, wahrnimmt und denkt, als wäre es das andere.

Empathie heisst in einer modernen Übersetzung Einfühlung in Anlehnung an das bedeutungsähnliche Wort Sympathie. Das griechische Empatheia bedeutete ursprünglich eine intensive Gefühlsregung. Die spätgriechische Bedeutung lautet jedoch «heftige Leidenschaft». Der Begriff der Empathie wird im deutschen Sprachraum seit Ende der 1960er Jahre von Psychologen, Therapeuten, Seelsorgern, Ärzten, Linguisten und Soziologen verwendet. In der Psychoanalyse deckt sich der Begriff der Empathie mit den Begriffen Einfühlungsvermögen und heftige Leidenschaft, die bereits Sigmund Freud definiert hatte.

Schon 1906 hat sein Schüler Theodor Lipps Empathie so genau bestimmt, dass es mit dem heutigen Begriff deckungsgleich ist. Dabei unterschied er drei verschiedene Stufen:

- Die erste Ebene beinhaltet die generelle Empathie. Sie besteht dann, wenn die Form eines Objekts eine Aktivität hervorruft. Diese erste Stufe ist bereits auch in der Tierwelt nachgewiesen.
Was passiert eigentlich in der Phylogenese und in der Hirnforschung bei Empathie? 1996 ermöglichte es der Zufall einer italienischen Forschergruppe, in eine neue Dimension vorzustossen. An der Universität Parma studierte der Physiologie Professor Giacomo Rizzolatti an Schimpansen, wie zielgerichtete Handlungen geplant und ausgeführt werden. Dabei wurden die Gehirne der Tiere unter Narkose so extrem an einen Messfühler angeschlossen, dass diese im Wachzustand nicht schmerzten oder störten.

Jedesmal wenn ein Affe nun nach einer Nuss griff, wurde eine bestimmte Nervenzelle aktiviert, sandte ein bioelektrisches Signal aus und brachte das Messgerät zum Knattern. Aus einer Laune heraus streckte einer der Forscher seine eigene Hand nach der Nuss aus, und siehe da: Das mit dem Affenhirn verbundene Gerät knatterte wie zuvor. Die Wissenschaftler glaubten zuerst an einen technischen Fehler, mussten dann aber feststellen, dass alles mit rechten Dingen zugegangen war. Der Affe verhielt sich genauso, als versetze er sich in den Kopf des Menschen: Indem er die Bewegung des andern verfolgte, spiegelten seine eigenen Neuronen diese Handlung wider, als hätte er sie selber ausgeführt. Diese Spiegelneuronen, wie sie jetzt hiessen, wurden bald auch beim Menschen nachgewiesen. Diese Spiegelnervenzellen verändern sich beim Beobachten anderer Menschen, ausgelöst durch bestimmte Gefühle. Diese Verhaltensweisen reproduzieren, d.h. spiegeln sich; es entsteht also der gleiche Zustand bei einem selbst wie bei seinem Gegenüber. Hält man in

\section{«Bei Menschen, die in ihrer Kindheit wenig Zuwendung empfunden haben, sind die Spiegelneuronen verkümmert, also kaum aktiv.»}

- Auf der zweiten Ebene vollzieht sich die natürliche Empathie. Hier ruft ein Objekt eine Aktivität hervor, die versucht, es in einen realen Kontext bzw. einen kausalen Zusammenhang einzuordnen. Auf dieser Ebene geschieht es, dass Objekte «vermenschlicht» werden.

- Auf der dritten, der höchsten Ebene der Empathie, reagieren wir auf echten menschlichen Ausdruck wie Gesten, Mimik und Stimmlagen.
Gesprächen mit anderen Menschen Augenkontakt, bringt die spiegelbildliche Mitaktivierung die entsprechenden Programme in einem selbst in Gang. Damit konnte das Phänomen der Gefühlsübertragung nach C. G. Jung wissenschaftlich begründet werden. Bei Menschen, die in ihrer Kindheit wenig Zuwendung empfunden haben, sind die Spiegelneuronen verkümmert, also kaum aktiv. Dasselbe gilt auch für Menschen mit Autismus. Auch bei «kopflas- 

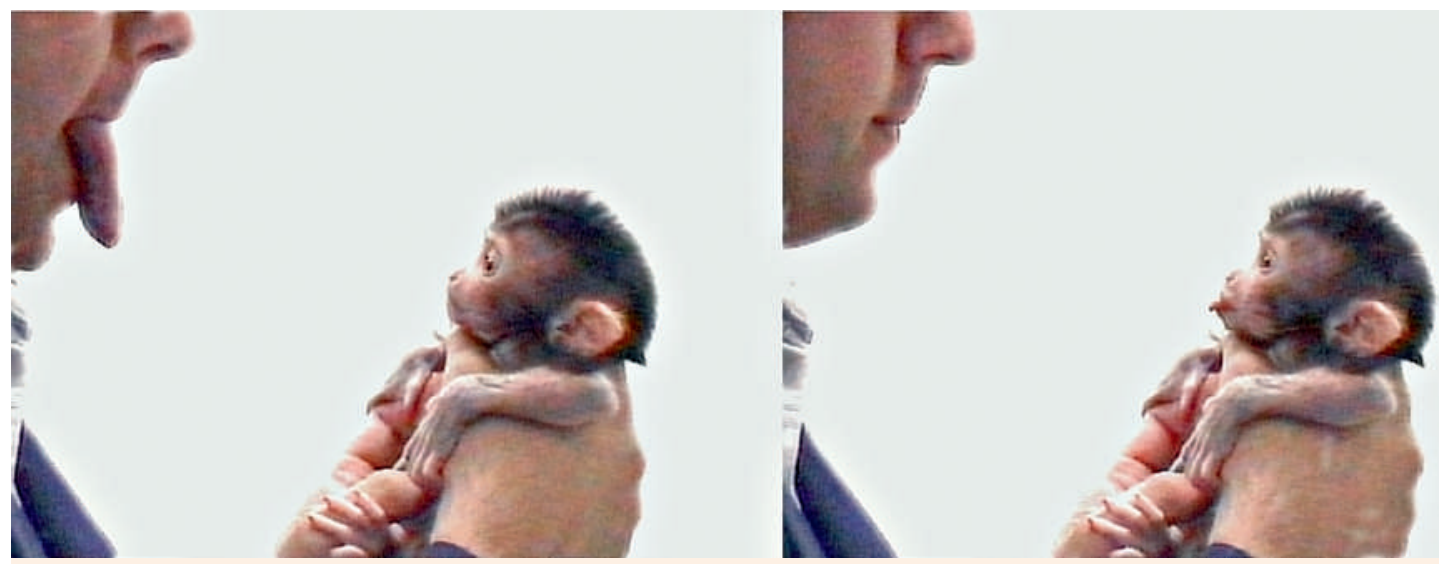

Ein Naturtalent! Der neugeborene Makake imitiert die Gesichtsausdrücke des Forschers. Möglich machen das Spiegelneuronen. Es sind Nervenzellen, die im Gehirn von Primaten beim reinen Betrachten eines Vorgangs das gleiche Aktivitätsmuster aufweisen wie beim aktiven Ausführen dieses Vorgangs.

(@) Gross L, PLoS Biology Vol. 4/9/2006, e311 http://dx.doi.org/10.1371/journal.pbio.0040311)

tigen Menschen» ist die Funktion der Spiegelneuronen wie bei den Autisten unzureichend. Dementsprechend werden diese Personen oft als weniger empfindsam, ja als überheblich und egozentrisch empfunden. Sie wirken häufig arrogant und abweisend. Glücklicherweise kann die Fähigkeit zur Empathie aber jederzeit aktiviert und verbessert werden.

Dass auch Mäuse über Einfühlungsvermögen verfügen, konnten kanadische und amerikanische Forscherteams nachweisen.

Der Niederländer Frans de Waal deckte den Irrtum der lateinischen Sentenz homo homini lupus auf: Früher war man der Auffassung, dass der Mensch und erst recht das Tier von Natur aus asoziale Bestien und, gemäss Thomas Hobbes, einem englischen Philosophen des 18. Jahrhunderts, nur durch sozialen Zwang im Zaum zu halten seien. Der in Atlanta lebende Verhaltensforscher führte verschiedene Beispiele auf:

- Rhesusaffen, die sich z. B. durch Ziehen an einer Kette Nahrung verschaffen konnten, hörten damit auf, wenn dadurch einem Artgenossen ein Stromstoss versetzt wurde. Sie hungerten lieber, als ihm Schmerz zuzufügen.

- Bei einem anderen Versuch liess sich ein Schimpanse nicht durch Drohungen oder Strenge von einem Dach locken, wohl aber durch einen Appell an sein Mitgefühl. Wenn die ihm vertraute Forscherin schluchzte, eilte er sofort herbei.

Die menschliche Fähigkeit zu Empathie, zu sozialem und moralischem Verhalten ist dementsprechend ein Teil unseres evolutionären Erbes.

\section{Untersuchungen zur Rolle der Empathie in der Medizin}

In der klinischen medizinischen Forschung waren es vor allem S. W. Mercer und W. Reynolds im Jahr 2002, welche die ärztliche Empathie als emotiona- les Element in der Arzt-Patienten-Interaktion sahen.

To understand a patient's situation, perspective and feelings.

To communicate that understanding and check its accuracy.

To act on that understanding with the patient in helpful therapeutic way.

Operationalisiert wurde diese Definition ärztlicher Empathie in der Skala Consultation and Relational Empathy (CARE), welche die ärztliche Empathie aus der Perspektive des Patienten erfasst. Die Items der CARE-Skala spiegeln verschiedene konkrete Verhaltensweisen ärztlicher Empathie wider, wie sie im Gespräch und Kontakt mit den Patienten auftreten. Dazu gehören z. B.:

- Hat der Arzt Sie Ihre eigene Krankheitsgeschichte erzählen lassen? Hatten Sie genug Zeit, Ihre

\section{Zeitmangel und Stress wurden als die schlimmsten Empathiekiller genannt.}

Krankheit ausführlich zu beschreiben? Hat er Sie dabei nicht unterbrochen oder abgelenkt?

- Hat der Arzt Ihnen wirklich zugehört? Hat er dem, was Sie gesagt haben, seine volle Aufmerksamkeit geschenkt und dabei nicht auf seine Unterlagen oder auf den Computer geschaut?

- Hat der Arzt Ihnen geholfen, einen Weg zu finden, mit Ihrer Krankheit umzugehen? Hat er gemeinsam mit Ihnen erkundet, wie Sie selbst Ihren Gesundheitszustand verbessern können? Hat er Sie ermutigt, anstatt Sie zu belehren?

Praktische Untersuchungen haben gezeigt, dass im Durchschnitt Ärzte ihre Patienten nach 60 Sekun- 
den unterbrechen, weil sie glauben, bereits zu wissen, worum es geht. Einmal unterbrochen, bringen nur noch zwei Prozent aller Patienten ihre Schilderung zu Ende.

Nachforschungen haben weiter ergeben, dass wenn sich Ärzte empathisch zu ihren Patienten verhalten, das patient enablement, d.h. die durch die ärztliche Behandlung und Kommunikation erzielte Befähigung des Patienten, mit der aktuellen Krankheitssituation umgehen zu können, verbessert werden kann. Dies gilt vor allem auch für onkologische Patienten. Mit dieser Empathie kann so der emotionale Distress reduziert und die Lebensqualität erhöht werden. In eindrücklicher Weise zeigte die Psychologin Melanie Neumann 2010 auf, dass dieses Verhalten auch in der Rehabilitation von grosser Bedeutung ist. Befragte Ärzte formulierten klar, welch grossen Stellenwert die Bedeutung der Empathie in der Behandlung ihrer Rehabilitationspatienten hat. Sie wiesen aber auch auf verschiedene Barrieren hin, die meistens ihren Ursprung in der Arbeitsintensität und in den organisatorischen Rahmenbedingungen haben. Zeitmangel und Stress wurden durchs Band als die schlimmsten Empathiekiller genannt, nur als Beispiel etwa die Fallpauschale, die Patienten zu Fällen macht und die individuelle Betreuung nicht berücksichtigt.

Wir älteren Ärzte erinnern uns noch gut, dass bis in die siebziger Jahre der "Gott in Weiss» das Mass aller Dinge war. Der weltberühmte Kinderarzt Guido Fanconi z.B. entlöhnte seine Assistenzärzte nicht und begründete dies so: «Mein Name sei ihr Lohn.»

Wie damals Patientenvorstellungen z. T. abliefen, beschreibt Oswald Oelz in «Antreten zur Visite» 2010 wie folgt. Schauplatz: eine Vorlesung im Wintersemester 1971 an der Universität Zürich:

«Im grossen Hörsaal dozierte der in jeder Hinsicht mächtige Chef der Urologischen Klinik. Nacheinander stellte er fünf junge Männer vor, die an einem Hodentumor litten. Mit jedem ging er väterlich um, vermittelte den Eindruck, alles stehe zum Besten, und liess sie dann wieder aus dem Raum führen. Als sich die Türe hinter dem Letzten geschlossen hatte, sagte der Professor: «Meine Damen und Herren, alle diese Herren werden in sechs Monaten tot sein.»

Glücklicherweise hat die Medizin in der Diagnostik und Behandlung auch äusserst bösartiger Krankheiten grosse Fortschritte gemacht. «Wir erhalten durch Labortest, Computertomographie und andere technische Hilfsmittel innert weniger Stunden präzise Diagnosen. Die technischen Fortschritte in der Diagnostik bringen innert weniger Stunden differenzierte Diagnosen. Aber wir erfahren wenig über das Befinden und die Ängste der Patienten.» (Oswald Oelz).

Auch wird der Patient heute nicht mehr angelogen. Er wird informiert und entscheidet zusammen mit dem Behandlungsteam über Art und Weise und
Ausmass von Behandlung und Pflege. Der medizinische Fortschritt basiert zu einem grossen Teil auf der Spezialisierung: Auf einem immer kleineren Gebiet kann und weiss der Arzt immer mehr. Der auf einzelne Organe spezialisierte Mediziner hat weniger den ganzen Menschen im Auge als der Hausarzt und der klassische Generalist. Oswald Oelz bringt das Beispiel von Franz Ingelfinger, einem der gescheitesten und einflussreichsten Mediziner der USA, der selber an Speiseröhrenkrebs erkrankte. Als dies bekannt wurde, waren sofort zahllose Topspezialisten mit Ratschlägen zur Stelle. Schliesslich war selbst dieser so kluge und informierte Patient völlig verwirrt, bis ihm ein Freund riet: «Weisst Du, was du brauchst, Franz? Du brauchst einen Doktor!». Ingelfingers Hausarzt nahm die Fäden in die Hand, und der Patient überlebte fünf Jahre.

Das alte Idealbild vom Arzt, der jahraus, jahrein aufopfernd für seine Patienten verfügbar ist, ist sicher passé. Chefärzte verbringen einen grossen Teil ihrer Zeit an diktierten Sitzungen. Die beispielhafte Patientenbetreuung und Demonstration der ärztlichen Kunst wird dadurch sekundär. Auch Kaderund Assistenzärzte, sogar Pflegepersonen verbringen immer mehr Zeit mit Leistungserfassung am Computer statt mit Leistungserbringung am Patienten. Aus Beschreibungen junger Ärzte über ihre Weiterbildungszeit möchte ich hier das Beispiel eines jungen Arztes anführen, der sich für ein Medizinstudium entschied, weil er Gutes für die Menschen tun wollte und ihn die Aura des Arztberufes faszinierte. Er erlebte aber eine grosse Enttäuschung. Er dachte, Empathie sei das Wichtigste in diesem Beruf, musste dann aber feststellen: «Dafür ist im Spitalalltag nur wenig Platz. Um die Patienten kann ich mich kaum

\section{«Empathie ist der Katalysator für ein erfolgreiches ärztliches Handeln.»}

kümmern. Es herrscht eine Kultur des Egoismus und der Missgunst.» Er zitierte dabei den Chefarzt, der einmal zu ihm sagte: «In der Ausbildung geht es mir vor allem darum, Ihre Frustrationstoleranz zu erhöhen.» Verständlich ist deshalb auch, dass es gerade Frauen oft schwer fällt, eine solche entsprechende Weiterbildung durchzustehen.

Leider wird schon früh der Zugang zur Ausbildung zum Arzt sehr schwierig gemacht. Seit 1998 gilt für ein Medizinstudium in der Schweiz der Numerus Clausus: Zum Studium an den Universitäten Zürich, Bern, Basel oder Freiburg wird nur zugelassen, wer einen entsprechenden Eignungstest besteht. Unterdessen hat sich ein eklatanter Mangel an einheimischem Nachwuchs entwickelt. Wäre unser Gesund- 
heitssystem überhaupt noch durchführbar ohne ausländische Ärzte? Bis vor kurzem waren z. B. in einem mir bekannten Regionalspital sämtliche Assistenzärzte Deutsche. Unterdessen hat aber Deutschland selber einen grossen Ärztemangel, es holt nun Ärzte aus dem Osten Europas, aus Asien und Afrika. Auch wir haben nun immer mehr Fachpersonal aus diesen Ländern. Nicht etwa, dass diese Ärzte eine schlechtere Ausbildung hätten, nein, das Hauptproblem liegt in der Verständigung. Es ist nicht mehr möglich, eine exakte Anamnese in der Muttersprache unserer Patienten zu erheben. Also kann auch die Empathie nicht spielen, die bei diversen Kontakten zwischen Arzt und Patient so nötig wäre. Das ist ein ernstzunehmendes Problem, vor allem auch im Hinblick darauf, dass jetzt schon und in Zukunft sowieso der hausärztliche Notfalldienst immer mehr am Spital gewährleistet werden soll. Kann dies wirklich das Ziel unserer medizinischen Versorgung sein?

Professor Hans Stalder hat kürzlich in der Schweizerischen Ärztezeitung einige markante Sätze formuliert: «Selbstverständlich schätzen die Patienten kompetente Ärzte, die eine messbare und quantifizierbare Medizin betreiben. Allerdings belegen praktisch alle Studien zur Patientenzufriedenheit, dass Patienten vor allem die «Menschlichkeit» des Arztes schätzen - Empathie und Einfühlsamkeit, Kommunikation. Genügend Zeit für die Konsultation, unkomplizierter Zugang zur Praxis und Kontinuität der Behandlung sind weitere wichtige Wünsche. Dazu braucht es jedoch eine Fähigkeit zur Kommunikation.»

Lassen Sie mich mit einigen Gedanken des Theologen und Philosophen Pater Albert Ziegler schliessen: Er hat in einer ausführlichen Publikation zum Thema «Altenpflege und Geriatrie - Prüfstein der ärztlichen Kommunikation» ausgeführt, dass man bei der Begegnung mit dem Patienten den Grundsatz der menschlichen Begegnung beachten muss. Dieser Grundsatz heisst: «Menschen muss man mögen. Das bedeutet: Menschen muss man mögen, auch wenn sie in Tat und Wahrheit nicht so sind, wie man sie sich wünscht. Das heisst noch genauer: Menschen muss man mögen - so dumm wie sie sind. Denn es gibt keine anderen.» In seinen markanten und eindringlichen Formulierungen erwähnt er auch, dass man das Gute beim andern sehen muss, auch wenn es klein ist. «Entdecke das Gute im andern, auch wenn Du lange suchen musst. Jeder hat verborgene Ressourcen. (...)

Verschweige das Ungute nicht und kehre es nicht unter den Teppich. Doch hilf dem andern, es beim nächsten Mal besser zu machen. Zeige ihm, wie es geht. Gehe ihm mit dem guten Beispiel als Vorbild voran. Sprich darum den andern zunächst dort an, wo er besser ist als Du, dann hast du ihm Aufmerksamkeit gewidmet, Wohlwollen geschenkt und seine Würde gewahrt. Dies hat nicht mit Anbiederei oder Kumpelhaftigkeit zu tun, wohl aber mit jener verstehenden Konsequenz, die Festigkeit und Güte verbindet. Damit macht sich das alte Wort auch im ärztlichen Alltag wahr: ‘Fortiter in re; suaviter in modo». (...) Im Grundsätzlichen wahr, aber behutsam in der Weise des Vorgehens.»

Lassen Sie mich zusammenfassen:

- Empathie ist älter als die Medizin.

- Medizin ohne Empathie ist eine leere Worthülse.

- Empathie ist in der heutigen modernen Medizin voller Apparate und biotechnologischer Therapie kein Luxus.

- Empathie ist der Katalysator für ein erfolgreiches ärztliches Handeln.

- «It's nice to be important, but it is even more important to be nice. (...) The good physician treats the disease; the great physician treats the patient, who has the disease.» (Sir William Osler)

\section{Weiterführende Literatur}

De Waal F. Das Prinzip Empathie. München: Carl Hanser; 2011. 